\title{
New treatment options in the management of fibromyalgia: role of pregabalin
}

\author{
Grazyna Zareba \\ Department of Environmental \\ Medicine, University of Rochester, \\ School of Medicine and Dentistry, \\ Rochester, NY, USA
}

Correspondence: Grazyna Zareba Department of Environmental Medicine, University of Rochester School of Medicine and Dentistry, 575 Elmwood Ave., Box EHSC, Rochester, NY 14642, USA

Tel +I 5852750795

Fax + I 585256259 |

Email grazyna_zareba@urmc.rochester.edu

\begin{abstract}
Fibromyalgia (FM) is a common, chronic pain disorder with unknown etiology, characterized by widespread musculoskeletal pain and tenderness, and accompanied by several other symptoms such as sleep disturbance, fatigue, and mood disorders. Pregabalin is the first drug approved for the treatment of FM. Pregabalin has analgesic, anticonvulsant, and anxiolytic activity and has earlier demonstrated efficacy in the management of neuropathic pain associated with diabetic peripheral neuropathy, postherpetic neuralgia, and as adjuvant therapy for adult patients with partial onset seizures. Pregabalin, a lipophilic gamma-aminobutyric acid (GABA) analog, is $\alpha_{2} \delta-1$ ligand that binds to, and modulates, voltage-gated calcium channels. This modulation is characterized by a reduction of the excessive neurotransmitter release that is observed in certain neurological and psychotic disorders. Several randomized, double-blind, placebo-controlled studies have demonstrated that pregabalin has been effective in pain management, improving sleep quality and fatigue, as well as in several domains of health related quality of life. Because of mild to moderate adverse effects it can be considered a well-tolerated therapy for FM.
\end{abstract}

Keywords: fibromyalgia, pregabalin, GABA analog, fibromyalgia treatment

\section{Introduction}

Fibromyalgia (FM) is a common, chronic pain disorder with unknown etiology, characterized by widespread musculoskeletal pain and tenderness. It has a prevalence of about $2 \%$ in the US population (Wolfe et al 1995) and $1 \%$ to $11 \%$ in other countries (McBeth and Jones 2007), and it is more common in adult women than in men, with prevalence of $3.4 \%$ vs $0.5 \%$, respectively (Wolfe et al 1995). The prevalence of FM increase with age, $(>7.0 \%$ in women) with highest values observed between 60 and 79 years (Pongratz and Sievers 2000). Clinical studies have demonstrated that the anti-seizure drug pregabalin is effective in the treatment of FM and its related symptoms (Lyrica $^{\circledR}$; Owen 2007). Pregabalin (Lyrica ${ }^{\circledR}$; Pfizer Inc.) has been approved in 2007 by the US Food and Drug Administration (FDA) as the first drug for the treatment of fibromyalgia, effective in reducing symptoms of pain, disturbed sleep and fatigue. Pregabalin (S-isobutyric gamma-aminobutiric acid, Figure 1), a second generation anticonvulsant, is a gamma-aminobutiric acid (GABA) analog with a similar structure and mechanism of action to gabapentin. It is a pharmacologically active S-enantiomer of racemic 3-isobutyl GABA which has been developed as a follow-up compound to gabapentin, an agent with proven antiepileptic, analgesic and anxiolytic activity (Lyrica $\left.{ }^{\circledR}\right)$.

Pregabalin has been approved earlier by the FDA for the treatment of neuropathic pain associated with diabetic peripheral neuropathy, postherpetic neuralgia and as adjunctive therapy for refractory partial seizures (Frampton and Foster 2005; Gray 2007; Zareba 2007). Pregabalin has also proven efficacy in adjunctive therapy of generalized anxiety disorder, social anxiety disorder and acute pain 


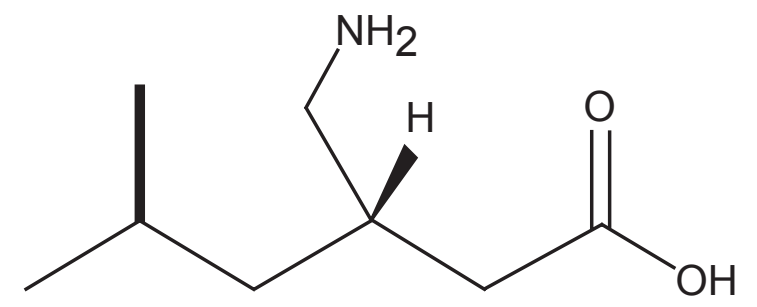

Figure I Chemical structure of pregabalin.

$\left(\right.$ Lyrica $^{\circledR}$; MICROMEDEX ${ }^{\circledR}$ ). This review focuses on the pharmacology, mechanism of action, and clinical studies of pregabalin in FM therapy.

\section{Fibromyalgia}

According to the criteria published in 1990 by the American College of Rheumatology, FM is defined as widespread pain of at least 3 months' duration and pain on palpation of at least 11 of 18 specific tender sites on the body (Merck; Wolfe et al 1990). Pain, fatigue, and sleep disturbance are observed in all patients (Mease 2005). Additional features of FM include stiffness, skin tenderness, postexertional pain, irritable bowel syndrome, cognitive disturbances, overactive bladder syndrome or interstitial cystitis, tension or migraine headaches, dizziness, fluid retention, paresthesias, restless legs, Raynaud's phenomenon, and mood disturbances (Bennet 1999; Mease 2005). FM is strongly associated with additional symptoms such as anxiety and depressive syndromes, chronic fatigue syndrome, myofascial pain syndrome, hypothyroidism, and many of the inflammatory rheumatic diseases (Aaron et al 2000; Mease 2005). Several studies suggest that neurotransmitter function deregulation, particularly serotonin, norepinephrine, and substance-P, and an abnormality of sensory processing within the central nervous system are involved in the pathophysiology of FM (Bennet 1999; Mease 2005). The diagnosis is made clinically, with no laboratory or radiological tests available. In clinical practice FM is difficult to diagnose since many symptoms characteristic for FM are also observed in other patients with chronic pain. For example, $80 \%$ of patients with FM also fulfill criteria for chronic fatigue syndrom (Aaron and Buchwald 2001). Several studies have demonstrated that many patients with FM suffer significant disability and reduced quality of life (Mease 2005; Rooks 2007; Wu et al 2007).

In FM patients, altered pain processing as evidenced by brain imaging and a 3-fold higher concentration of cerebrospinal fluid substance $\mathrm{P}$ has been demonstrated
(Russell et al 1994; Goldenberg 2007). A genetic and neuroendocrine predisposition has also been reported. In first degrees relatives of FM patients, an approximate 8-fold greater risk of developing FM has been observed (Arnold et al 2004a). Specific polymorphisms in the serotonin transporter gene and the catechol-O-methyltransferase (enzyme inactivating catecholoamines) have been associated with FM occurrence (Cohen et al 2002; Gursay et al 2003). The lower pain threshold in FM patients involves a complex variety of neural pathways and neurotransmitters (ie, serotonin, norepinephrine), especially those critical to pain inhibition (Staud 2006; Goldenberg 2007; Crofford 2008).

In clinical trials on FM, pain has been the most common outcome measure and it has been generally evaluated with $100 \mathrm{~mm}$ numerical rating scales (NRS), visual analog scales (VAS), or the brief pain inventory (BPI) (Goldenberg 2007; Rooks 2007). Body function is assessed by self-reported, validated instruments, most often the fibromyalgia impact questionnaire (FIQ) which is a 10-item, reliable measure of physical function, common fibromyalgia symptoms and general well-being over the previous 7 days (Bennett 2005). Short-Form 36 Health Survey (SF-36) is increasingly used as a valid and reliable, self-administered measure of physical and emotional function, and quality of life in FM patients (Ware and Sherbourne 1992; Kosinski et al 1999).

Treatment of FM is symptom-based in order to alleviate pain, restore sleep and improve physical status. It has been demonstrated that many patients with FM benefit from a multidisciplinary approach in clinical practice (Goldenberg et al 2004; Arnold 2006; Rooks 2007). Current pharmacological treatment guidelines, such as those described by the American Pain Society (Burckhard et al 2005), recommend tricyclic antidepressants, analgesics, muscle relaxants, benzodiazepines, nonsteroidal anti-inflammatory agents, hypnotics, corticosteroids, opiates, and soft tissue injections of topical anesthetics (Goldenberg et al 2004; Mease 2005; Goldenberg 2007; Crofford 2008). Most of these have demonstrated effectiveness in reducing pain, but few have been shown to be effective in improving the other major symptoms of FM, such as fatigue, sleep disturbances, and mood abnormalities. Clinical studies provide evidence that tricyclic antidepressants are the most effective in FM treatment but their limitations are mostly due to relatively narrow therapeutic index and poor tolerability. Better tolerated selective serotonin reuptake inhibitors (SSRIs; fluoxetine) and dual serotonin-norepinephrine 
reuptake inhibitors (SNRIs; milnacipran, duloxetine) have demonstrated moderate effectiveness (Goldenberg 2007). Tramadol is the only analgesic shown to be helpful in FM treatment (Biassi 1998; Goldenberg 2007).

Nonpharmacologic treatments showing beneficial effects in relieving some symptoms of FM include cognitive behavioral therapy, exercise (strength and flexibility training), improvement of sleep-related disturbances, and complementary and alternative medicine, ie, osteopathic manipulations, acupuncture, low-power laser therapy, and balneotherapy (Arnold 2006; Rooks 2007).

According to Staud et al (2006), three important strategies for FM therapy appear useful at this time: 1) reduction of peripheral nociceptive input, particularly from muscles (through physical therapy, muscle relaxants, muscle injections, and anti-inflammatory analgesics); 2) improvement or prevention of central sensitization (through cognitive behavioral therapy, sleep improvement, NMDA receptor antagonists, and anti-seizure medications); and 3) treatment of negative affect, particularly depression (through pharmacological and behavioral treatment).

Pregabalin represents a new option in FM treatment due to its higher spectrum of action showing efficacy in reducing pain and improvement of other accompanying symptoms.

\section{Pregabalin Mechanism of action}

Pregabalin has proven analgesic, anticonvulsant, and anxiolytic activity. Multiple cellular effects have been proposed for its mechanism of action, including modest effects on the GABAnergic neurotransmitter system and on voltage-gated potassium channels (Freiman et al 2001; Sills 2006). However, a single common mechanism, inhibition of calcium currents via high-voltage-activated channels containing the $\alpha_{2} \delta-1$ subunit, leading in turn to reduced neurotransmitter release and attenuation of postsynaptic excitability is believed to have a predominate effect on its mechanism of action (McClelland et al 2004; Sills 2006). This effect has been consistently observed at therapeutically relevant concentrations in pre-clinical studies with pregabalin. Studies on mice with mutations leading to the substitution of arginine at position 217 with alanine on the $\alpha_{2} \delta-1$ subunit demonstrated this unit to be a target for pain control, and that pregabalin, by binding to this subunit, achieves its analgesic activity (Field et al 2006). Additional animal studies have shown pregabalin's anticonvulsant (Vartanian et al 2006) and anxiolytic activity (Field at al 2001).
It has been demonstrated that although pregabalin is a structural derivative of the inhibitory neurotransmitter GABA, it does not bind directly to $\mathrm{GABA}_{\mathrm{A}}, \mathrm{GABA}_{\mathrm{B}}$, or benzodiazepine receptors, does not augment $\mathrm{GABA}_{\mathrm{A}}$ responses in vitro, does not alter GABA concentration in the animal brain, or have acute effects on GABA uptake or degradation $\left(\right.$ Lyrica $\left.^{\circledR}\right)$. However, prolonged application of pregabalin increases the density of GABA transporter proteins and increases the rate of functional GABA transport. Pregabalin does not block sodium channels, is not active at opiate receptors, and does not alter cyclooxygenase enzyme activity. It is inactive at serotonin and dopamine receptors and does not inhibit dopamine, serotonine, or noradrenaline reuptake $\left(\right.$ Lyrica $^{\circledR}$; MICROMEDEX ${ }^{\circledR}$ ).

\section{Pharmacokinetics}

After oral administration, pregabalin is well absorbed with peak plasma concentrations after 1.5 hours and its bioavailability is approximately $90 \%$. The $\mathrm{C}_{\max }$ and AUC values are dose proportional. After repeated administration steady state is achieved within 24-48 hours. Food consumption does not affect absorption of pregabalin but it reduces $\mathrm{C}_{\max }$ by $25 \%-30 \%$ and increases $\mathrm{T}_{\max }$ to approximately 3 hours $\left(\right.$ Selak 2001; Lyrica $\left.^{\circledR}\right)$.

Pregabalin does not bind to plasma protein and its apparent volume of distribution after oral administration is approximately $0.5 \mathrm{~L} / \mathrm{kg}$. Pregabalin is a substrate for the system $L$ transporter responsible for transport of large amino acids. In animal studies it has been demonstrated that pregabalin crosses the blood-brain barrier. It has also been shown that pregabalin crosses the placenta and is present in milk of lactating animals (Selak 2001).

Metabolism in liver is negligible (Selak 2001; MICROMEDEX $^{\circledR}$ ). Renal excretion is the primary route of elimination as unchanged pregabalin $(92 \%-99 \%)$ and a minor metabolism to an N-methylated derivative $(0.9 \%)$ and an unidentified metabolite $(0.4 \%)$ (Willmore 2000). Elimination half-life is 6.3 hours and a mean renal clearance is $67.0-80.9 \mathrm{~mL} / \mathrm{min}$ in young healthy subjects. Pregabalin elimination is nearly proportional to creatinine clearance. In patients with renal dysfunction dose reduction is necessary and in patients on chronic hemodialysis, supplemental pregabalin may be required due to reduction in pregabalin plasma concentration by $50 \%$ (Randinitis et al 2003). No drug interactions with oral contraceptives, lorazepam, oxycodone, ethanol, phenytoin, carbamazepine, valproic acid, and lamotrigine were observed (Brodie et al 2005). Pregabalin absorption was unaffected by gabapentin coadministration, 
although a small reduction in the rate of absorption was observed (MICROMEDEX ${ }^{\circledR}$ ).

\section{Clinical efficacy studies}

In a multicenter, double-blind, placebo-controlled, 8-week randomized efficacy and safety clinical trial (Crofford et al 2005), the effects of pregabalin (administered at the doses of 150,300 , or $450 \mathrm{mg} /$ day) on pain, sleep, fatigue, and healthrelated quality of life were studied in 529 patients with FM. The comparison of end point mean pain scores from daily diary ratings of pain intensity between each of the pregabalin treatment groups and the placebo group revealed that pregabalin at $450 \mathrm{mg} /$ day significantly reduced the average severity of pain in the primary efficacy analysis as compared with placebo $(-0.93$ on a $0-10$ scale; $p \leq 0.001)$. The improvement in pain $\geq 50 \%$ at the end point was observed in patients from the same group (29\%, vs $13 \%$ in the placebo group; $p=0.003$ ). Pregabalin administration at doses 300 and $450 \mathrm{mg} /$ day was associated with significant improvements in sleep quality, fatigue, and global measures of change. Pregabalin at the dose of $450 \mathrm{mg} /$ day improved several domains of health-related quality of life. End point scores were significantly higher than those obtained in the placebo group in social functioning, bodily pain, vitality, and general perception in the SF-36 Health Survey. Most adverse events were mild or moderate with the most frequently observed being dizziness and somnolence. Rates of discontinuation due to dizziness and somnolence were similar across all 4 treatment groups. Non-central nervous system adverse events including weight gain (no withdrawal) and peripheral edema (1 patient) with no changes in cardiovascular or renal function were observed.

FM is frequently associated with anxiety and depressive symptoms which are reported by approximately $1 / 3$ of patients (Wolfe et al 1995; White et al 2002; Antai-Otong 2005). Arnold et al (2007) assessed the prevalence and severity of anxiety and depressive symptoms in FM patients, using the patient-reported Hospital Anxiety and Depression Scale in a previously described cohort (Crofford et al 2005). In order to determine the impact of these symptoms on response to pain reduction after the treatment of patients with pregabalin $(\mathrm{N}=529)$, the association between improvements in anxiety and depression and pain relief were evaluated. It has been shown that significantly more patients reported anxiety symptoms (71\%) than depressive symptoms (56\%, $\mathrm{p}<0.0001)$. Much of the pain reduction appeared to be independent of improvements in anxiety or mood symptoms. Pregabalin reduced pain in patients with or without anxiety or depressive symptoms, suggesting that pregabalin would be efficacious in patients regardless of these symptoms.

Pregabalin has also been studied in combination therapy of FM. Calandre et al (2007) conducted an openlabel, 12-week study in 19 female FM patients currently being treated with quetiapine $(76 \mathrm{mg} /$ day $)$. Pregabalin was introduced at a starting dose of $75 \mathrm{mg}$ /day which was subsequently adjusted according to the drug's efficacy and tolerability (range 75-300 mg/day). Outcome measures included the FIQ, the Pittsburgh Sleep Quality Index, the Beck Depression Inventory, the State and Trait Anxiety Inventory, and the SF-12 Health Survey. Pregabalin significantly improved the pain and tiredness after awakening subscales of the FIQ as well as the physical component of the SF-12. Out of 6 patients who withdrew from the study, 3 did it because of side effects. Studies demonstrated that the use of combined therapy of pregabalin and quetiapine can be a useful augmentation strategy in the treatment of fibromyalgia patients.

In the recently published multicenter, double-blind, placebo-controlled randomized trial on pregabalin's efficacy and safety, 748 patients with FM received placebo or pregabalin at doses of 300,450 , or $600 \mathrm{mg} / \mathrm{day}$, twice daily for 13 weeks (Mease et al 2008). The evaluation of symptomatic relief of pain associated with FM was based on comparison of endpoint mean pain scores between each pregabalin group and placebo. Another outcome variable, management of FM, included endpoint mean pain scores, Patient Global Impression of Change (PGIC), and the FIQ -Total Score. Secondary outcomes included assessments of sleep, fatigue, and mood disturbance. In all pregabalin treated patients statistically significant improvement in endpoint mean pain score and in PGIC response was observed as compared with placebo. Statistically significant improvement in assessments of sleep, the MOS-Sleep Disturbance, MOS-Sleep Quantity subscale, Sleep Problem Index, and in patients' impressions of their global improvement was observed in all pregabalin treatment groups. The most frequently reported adverse events were dizziness, somnolence, headache, infection, and weight gain. All these effects were of mild or moderate intensity. Dizziness and somnolence were the adverse effects that most frequently led to withdrawal among pregabalin treated patients ( $9 \%$ and $6 \%$, respectively). A dosage related increase in patient's withdrawal due to any adverse effects $(11 \%, 19 \%, 22 \%$, and $33 \%$ for placebo, 300,450 , and $600 \mathrm{mg} /$ day pregabalin groups, respectively) was observed. Sixty-six patients (11.8\%) experienced a clinically significant increase in body weight of $7 \%$ or 
more from baseline to end of treatment; however, none of the patients with weight gain $\geq 7 \%$ discontinued treatment prematurely.

A long-term, multicenter, double-blind, placebo-controlled, randomized discontinuation trial (FREEDOM) demonstrated the durability of pregabalin effects for relieving FM pain in patients who responded to the treatment (Crofford et al 2008). The trial, performed in adults with FM and $\geq 40-\mathrm{mm}$ score using 100-mm VAS, included a 6-week open-label pregabalin treatment phase $(\mathrm{N}=1051)$ followed by 26 -week, double-blind treatment phase with placebo or pregabalin. During the open label weeks $1-3$, patients received escalating dosages of pregabalin to determine their optimal dosages, and during open label weeks 4-6, patients received their optimal fixed dosages (300, 450, $600 \mathrm{mg} /$ day). To be randomized, patients must have had $\geq 50 \%$ decrease in pain VAS and a self-rating of "much" or "very much" improved on PGIC at the end of the open label period. In the double-blind treatment phase patients received placebo or the patient's optimal fixed dosage of pregabalin. Time to loss of therapeutic response (LTR), defined as $<30 \%$ reduction in pain (from open label baseline) or worsening of FM was a primary outcome. Out of all patients who entered the open label phase, 287 were randomized to placebo and 279 to pregabalin treatment. The observed time to LTR was longer for the pregabalin group as compared to placebo ( $\mathrm{p}<0.0001$ ). Kaplan-Meier estimates of time-to-event showed half the placebo group had LTR by Day 19 (Figure 2); half the pregabalin group still had not lost response by trial end. At the end of the double blind period, 174 (61\%) placebo patients met LTR criteria versus $90(32 \%)$ pregabalin patients. During the open label phase 178 patients $(17 \%)$ discontinued participation in the study due to treatment-related adverse events. During the double-blind phase $16 \%$ of pregabalin compared with only $7 \%$ of patients receiving placebo discontinued treatment due to adverse effects.

Another recently published study on the efficacy and safety of pregabalin monotherapy demonstrated that pregabalin is an important treatment option for FM patients (Arnold et al 2008). In a randomized, doubleblinded, placebo-controlled trial, 750 patients meeting American College of Rheumatology criteria for FM were randomly assigned to pregabalin $(300 \mathrm{mg} /$ day, $450 \mathrm{mg} / \mathrm{day}$, $600 \mathrm{mg} /$ day) or placebo, administered twice daily for

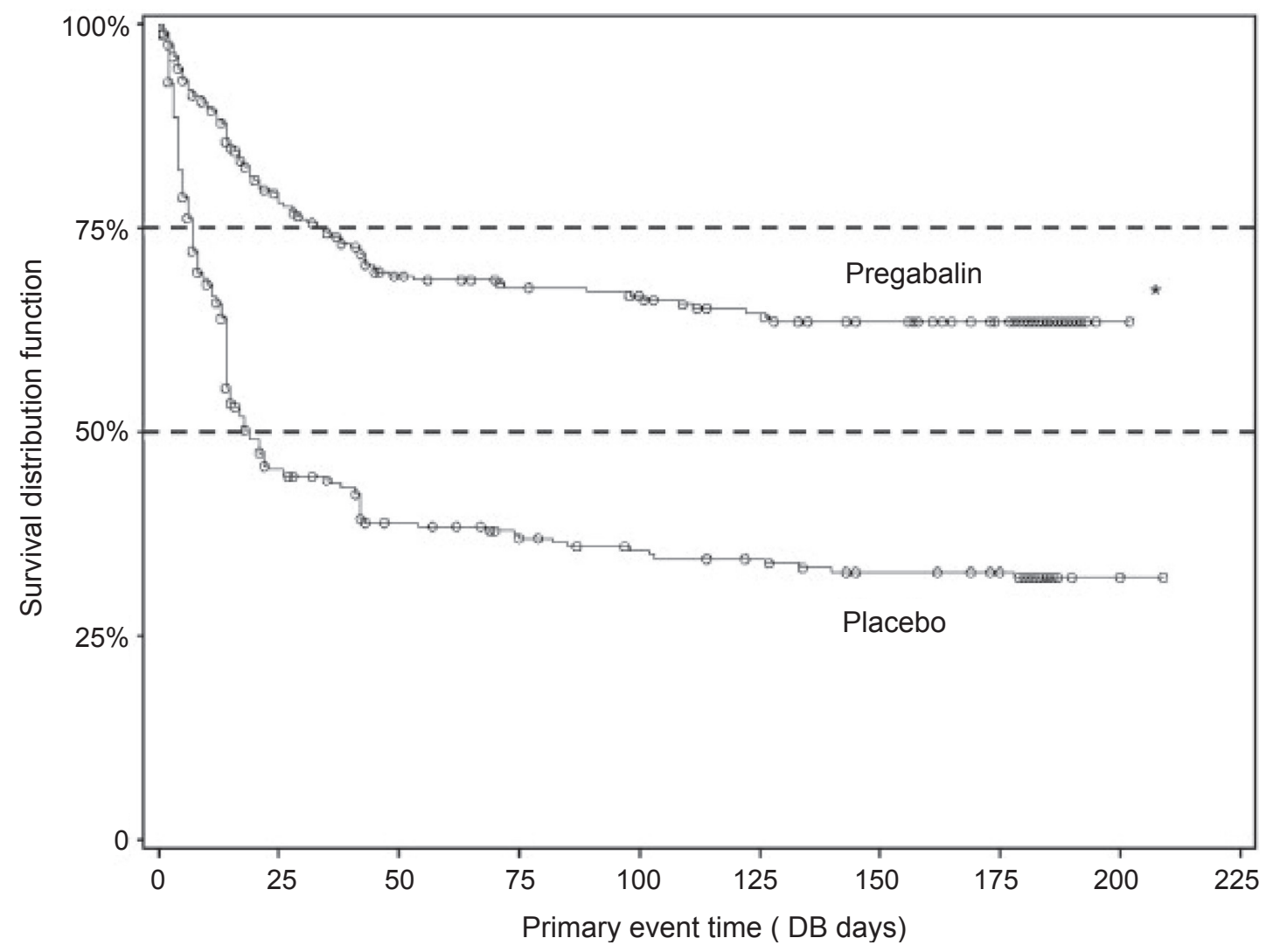

Figure 2 Kaplan-Meier plot of time to loss of therapeutic effect. Comparison (log-rank test) with placebo, $\mathrm{p}<0.000 \mathrm{I}$. Reproduced with permission from Crofford LJ, Mease PJ, Simpson SL, et al 2008. Fibromyalgia relapse evaluation and efficacy for durability of meaningful relief (FREEDOM): A 6-month, double-blind, placebo-controlled trial with pregabalin. Pain, 136:419-31. Copyright (C) 2008 Elsevier. 
14 weeks. The primary outcome variable was comparison of end point mean pain scores, derived from daily diary ratings of pain intensity ( $0-10$ scale), between each of the pregabalin groups and the placebo group. Mean changes in pain scores at the end point in pregabalin treated patients were significantly greater $(\mathrm{p}<0.001: 300 \mathrm{mg} /$ day, $0.71 ; 450 \mathrm{mg} /$ day $-0.98 ; 600 \mathrm{mg} /$ day, -1.00$)$ than in the placebo group. Pregabalin-treated patients reported improvement on PGIC ( $p<0.01$ for all 3 pregabalin doses) and significant improvements in total FIQ score for the $450 \mathrm{mg} /$ day $(p=0.004)$ and the $600 \mathrm{mg} /$ day $(p=0.003)$ doses. All 3 doses of pregabalin were associated with significant improvement in sleep. The most commonly reported pregabalin-related adverse events were dizziness and somnolence, which appeared to have been dose-related.

Gabapentin, a precursor of pregabalin, has been approved by the FDA for the treatment of postherpetic neuralgia and for adjunctive treatment of epilepsy, and it has also been evaluated in FM patients in a randomized, double-blind, placebo-controlled study (Arnold et al 2007). Gabapentin (1,200-2,400 mg/day) was administered to patients $(\mathrm{N}=75)$ for 12 weeks. Gabapentin-treated patients displayed a significantly greater improvement in the BPI average pain severity score $(\mathrm{p}=0.015$; estimated difference between groups at week $12=-0.92$ ). A significantly greater proportion of gabapentin treated patients compared with placebo-treated patients achieved response at the end point $(51 \%$ vs $31 \%$; $=0.014)$. Gabapentin compared with placebo also significantly improved the BPI average pain interference score, the Fibromyalgia Impact Questionnaire total score, the Clinical Global Impression of Severity, the Patient Global Impression of Improvement, the Medical Outcomes Study (MOS) Sleep Problems Index, and the MOS Short Form 36 vitality score, but not the mean tender point pain threshold or the Montgomery Asberg Depression Rating Scale. Gabapentin was generally well tolerated. However, as compared with placebo, a significantly higher incidence of sedation, lightheadedness, and dizziness was observed in gabapentin patients.

It should be pointed out that the primary outcome measure used in most clinical trials on FM is mean reduction in pain intensity and according to several authors, this approach does not adequately address the critical question of whether or not a statistically significant reduction in pain intensity versus placebo with a given treatment is clinically significant or meaningful (Mease 2005). Staud and Price (2008) pointed out that although an enrichment design applied in some studies on pregabalin (Mease et al 2008) can be efficacious clinically, the prior exposure to an experimental treatment in open-label qualification phase may invalidate drug-placebo comparisons made during a later randomized, double-blind phase.

\section{Safety and tolerability}

Adverse effects in clinical trials conducted under widely varying conditions varied for different disorders and have been described separately elsewhere $\left(\right.$ Lyrica $\left.^{\circledR}\right)$. In all premarketing trials of all populations combined, $14 \%$ of pregabalin-treated patients and $7 \%$ of placebo-obtaining patients discontinued prematurely due to adverse effects. In pregabalin patients, the observed withdrawal was mostly due to dizziness (4\%) and somnolence (3\%), whereas in the placebo group discontinuation due to the same adverse effects was $1 \%$ and $<1 \%$, respectively. In clinical trials of FM patients, $19 \%$ of patients treated with pregabalin (150-600 mg/day) discontinued treatment due to adverse reactions, mostly dizziness $(6 \%)$ and somnolence $(3 \%)$, whereas $10 \%$ of patients obtaining placebo withdrew due to the same adverse effects $\left(\right.$ Lyrica $\left.^{\circledR}\right)$.

The most frequently reported adverse effects of pregabalin in clinical trials of patients with FM were dose related and observed within $150-600 \mathrm{mg}$ /day vs placebo: dizziness $(23 \%-38 \%$ vs $9 \%)$, somnolence $(13 \%-22 \%$ vs $4 \%)$, headache $(11 \%-10 \%$ vs $12 \%)$, weight increase ( $8 \%-14 \%$ vs $2 \%$ ), fatigue ( $5 \%-8 \%$ vs $4 \%$ ), and balance disorder ( $2 \%-9 \%$ vs $0 \%)\left(\right.$ Lyrica $\left.^{\circledR}\right)$. These adverse effects were usually mild or moderate in severity. However, Mease et al (2008) reported recently a higher incidence of dizziness $(42 \%)$ and somnolence $(26 \%)$ which can indicate that patients with FM may be more sensitive to central nervous system related adverse effects. Non-central nervous system events observed more frequently in the pregabalin-treated patients included also peripheral edema. No changes in cardiovascular or renal function were observed in patients who developed peripheral edema. There were no clinically relevant differences in clinical laboratory evaluations, vital signs, physical examination findings, or electrocardiogram findings $\left(\right.$ Lyrica $^{\circledR} ;$ MICROMEDEX $\left.^{\circledR}\right)$.

\section{Other new developments in fibromyalgia treatment}

Several different pharmacological approaches have been undertaken in FM management. Besides described above clinical trials on $\alpha_{2} \delta-1$ ligands, selective SNRIs such as duloxetine and milnacipram demonstrate the biggest promise. Similarly to pregabalin, they reduce not only pain, the primary 
symptom of FM, but also improve other symptom domains, and some aspects of function and global assessments.

Duloxetine has been approved by the FDA for the treatment of major depressive disorder, generalized anxiety disorder, diabetic peripheral neuropathic pain and FM. A randomized, double-blind, placebo-controlled, multicenter study evaluated the efficacy and safety of duloxetine in subjects with primary FM (Arnold 2004b). Patients ( $\mathrm{N}=207,38 \%$ with current major depressive disorder) received duloxetine $60 \mathrm{mg}$ twice daily or placebo for 12 weeks. Duloxetine was an effective and safe treatment for many of the symptoms associated with FM in subjects with or without major depressive disorder, particularly for women, who had significant improvement across most outcome measures. Difficulty sleeping, oral dryness and constipation were the most frequently reported adverse effects in the duloxetine group.

Duloxetine was also studied in a 12-week, randomized, double-blind, placebo-controlled trial in 354 female patients with primary FM, with or without current major depressive disorder (Arnold 2005). Patients received duloxetine $60 \mathrm{mg}$ once daily $(\mathrm{N}=118)$ or twice daily $(\mathrm{N}=116)$, or placebo $(\mathrm{N}=120)$. Both duloxetine treated groups improved significantly on average pain severity score. The decrease of pain score was 55\%, $54 \%$, and 33\% (duloxetine once daily, duloxetine twice daily and placebo, respectively). The treatment effect of duloxetine on pain reduction was independent of the effect on mood and the presence of major depressive disorder. Patients treated with duloxetine had significantly greater improvement in BPI, pain severity and interference scores, FIQ, Clinical Global Impression of Severity, Patient Global Impression of Improvement, and several quality of life measures. The adverse effects were generally mild to moderate in severity with the most frequently observed being nausea.

Milnacipran, another selective SNRI has been approved in 1997 in Europe, Asia, and other countries for the treatment of depression but it is unavailable in the US. Milnacipram has been studied in 125 patients (98\% women) with FM (Gendreau et al 2005). In a double-blind dose escalation Phase II study, patients were randomly assigned in a 3:3:2 ratio to receive milnacipran twice daily, milnacipran once daily, or placebo for 3 months. Dose escalation to the target milnacipran dose of $200 \mathrm{mg}$ was achieved in $92 \%$ of twice daily and $81 \%$ of once daily participants. The twice daily milnacipran group showed statistically significant improvements in the weekly diary pain scores and more patients (37\%) reported a reduction in the weekly average pain scores by $\geq 50 \%$. In both once daily and twice daily milnacipram groups, improvements in global well being, fatigue, and other domains were observed. Response rates for patients receiving milnacipran were equal in patients with and without comorbid depression, but placebo response rates were considerably higher in depressed patients, leading to significantly greater overall efficacy in the nondepressed group. Milnacipran was generally well tolerated. Patient withdrawal due to adverse events was $14.4 \%$, with most commonly reported symptoms being headache and gastrointestinal problems (nausea, abdominal pain, constipation). Other adverse effects included orthostatic dizziness, hypertension, depression, lethargy, sweating, and hot flashes.

Clinical trials of SSRIs, fluoxetine (Wolfe et al 1994), and citalopram (Nørregaard et al 1995) have demonstrated mixed responses suggesting less consistent results than described above SNRIs (Arnold 2006).

Sedative hypnotics, such as sodium oxybate (sodium salt of gamma-hydroxybutyrate, precursor of GABA), used for the treatment of cataplexy and narcolepsy, have been also evaluated in FM treatment due to often observed sleep disturbances in FM patients (Russell 1999; Scharf et al 2003). However, a high risk of abuse, lethality at supratherapeutic doses or risk of hypnotic use, make usefulness of these drugs questionable (Griffiths and Johnson 2005).

Similar controversy may be associated with use of opiates. Tramadol, a novel analgesic with weak agonist activity at the mu opiate receptor combined with dual serotonin and norepinephrine reuptake inhibition, has been studied in several FM clinical trials (Biasi et al 1998; Bennett et al 2003). However, tramadol use has been associated with increased number of reports on abuse, dependency and presence of classic opioid withdrawal symptoms (Senay et al 2003).

Other new pharmacological approaches to FM treatment such as the use of 5-HT 3 antagonists (tropisteron) (Färber et al 2000; Späth et al 2004), NMDA antagonists (dextromethorphan) (Henriksson and Sorensen 2002; Staud et al 2005), or dopamine 3 receptor agonists (pramipexole) (Holman and Myers 2005) show some promising results. However, more studies are needed before their use can be considered for FM treatment.

\section{Conclusions}

FM is a chronic pain disorder which causes pain, fatigue, reduced quality of life, and disability. Although recent studies support the recommendation of a multidisciplinary approach in clinical practice, pharmacologic treatment remains the primary approach to management of patients with FM. Treatment goals include the improvement of 
symptoms, primarily pain and sleep, and the promotion of improvement of physical function, emotional well-being and other symptoms associated with FM. The complexity of FM makes it challenging for pharmaceutical companies to mount effective clinical trials to assess emerging therapies. Randomized controlled trials are generally difficult due to some factors such as a lack of understanding of the pathophysiology or heterogeneity of the FM patient population. However, results of several clinical studies have been already published and more are taking place to identify different potential therapeutic approaches. Ongoing clinical trials on newer agents seem promising, however the efficacy of all these agents has not been compared with pregabalin yet. Pregabalin offers several practical properties, such as lack of protein-binding and hepatic metabolism, and linear pharmacokinetics across its therapeutic range. Together with its demonstrated safety and tolerability, pregabalin represents an important new option for the treatment of FM.

\section{Disclosures}

The author reports no conflicts of interest.

\section{References}

Antai-Otong D. 2005. The art of prescribing. Depression and fibromyalgia syndrome (FMS): pharmacologic considerations. Perspect Psychiatr Care, 41:146-8.

Aaron LA, Burke MM, Buchwald D. 2000. Overlapping conditions among patients with chronic fatigue syndrome, fibromyalgia, and temporomandibular disorder. Arch Intern Med, 160:221-7.

Aaron LA, Buchwald D. 2001. A review of the evidence for overlap among unexplained clinical conditions. Ann Intern Med, 134:868-81.

Arnold LM. 2006. Biology and therapy of fibromyalgia. New therapies in fibromyalgia. Arthritis Res Ther, 8:212.

Arnold LM, Hudson JI, Hess EV, et al. 2004a. Family study of fibromyalgia. Arthritis Rheum, 50:944-52.

Arnold LM, Lu Y, Crofford LJ, et al. 2004b. A double-blind, multicenter trial comparing duloxetine with placebo in the treatment of fibromyalgia patients with or without major depressive disorder. Arthritis Rheum, 50:2974-84.

Arnold LM, Rosen A, Pritchett YL, et al. 2005. A randomized, double-blind, placebo-controlled trial of duloxetine in the treatment of women with fibromyalgia with or without major depressive disorder. Pain, 119:5-15.

Arnold LM, Goldenberg DL, Stanford SB, et al. 2007. Gabapentin in the treatment of fibromyalgia: a randomized, double-blind, placebo-controlled, multicenter trial. Arthritis Rheum, 56:1336-44.

Arnold LM, Crofford LJ, Martin SA, et al. 2007.The effect of anxiety and depression on improvements in pain in a randomized, controlled trial of pregabalin for treatment of fibromyalgia. Pain Med, 8:633-8.

Arnold LM, Russell IJ, Diri EW, et al. 2008. A 14-week, randomized, double-blinded, placebo-controlled monotherapy trial of pregabalin in patients with fibromyalgia. J Pain, 9:792-805.

Bennett RM. 1999. Emerging concepts in the neurobiology of chronic pain: evidence of abnormal sensory processing in fibromyalgia. Mayo Clin Proc, 74:385-98.
Bennett R. 2005. The Fibromyalgia Impact Questionnaire (FIQ): a review of its development, current version, operating characteristics and uses. Clin Exp Rheumatol, 23(Suppl 39):S154-62.

Bennett RM, Kamin M, Karim R, et al. 2003. Tramadol and acetaminophen combination tablets in the treatment of fibromyalgia pain: a doubleblind, randomized, placebo-controlled study. Am J Med, 114:537-45.

Bennett RM, Schein J, Kosinski MR, et al. 2005. Impact of fibromyalgia pain on health-related quality of life before and after treatment with tramadol/acetaminophen. Arthritis Rheum, 53:519-27.

Biasi G, Manca S, Manganelli S, et al. 1998. Tramadol in the fibromyalgia syndrome: a controlled clinical trial versus placebo. Int $J$ Clin Pharmacol Res, 18:13-9.

Brodie MJ, Wilson EA, Wesche DL, et al. 2005. Pregabalin drug interaction studies: lack of effect on the pharmacokinetics of carbamazepine, phenytoin, lamotrigine, and valproate in patients with partial epilepsy. Epilepsia, 46:407-13.

Burckhardt CS, Goldenberg D, Crofford L, et al. 2005. Guidelines for management of fibromyalgia syndrome pain in adults and children. American Pain Society (APS). Clinical Practice Guideline No.4:1-119.

Calandre EP, Morillas-Arques P, Rodriguez-Lopez CM, et al. 2007. Pregabalin augmentation of quetiapine therapy in the treatment of fibromyalgia: an open-label, prospective trial. Pharmacopsychiatry, 40:68-71.

Cohen H, Buskila D, Neumann L, et al. 2002. Confirmation of an association between fibromyalgia and serotonin transporter promoter region (5- HTTLPR) polymorphism, and relationship to anxiety-related personality traits. Arthritis Rheum, 46:845-7.

Crofford LJ. 2008. Pain management in fibromyalgia. Curr Opin Rheumatol, 20:246-50

Crofford LJ, Rowbotham MC, Mease PJ, et al. 2005. Pregabalin for the treatment of fibromyalgia syndrome: results of a randomized, doubleblind, placebo-controlled trial. Arthritis Rheum, 52:1264-73.

Crofford LJ, Mease PJ, Simpson SL, et al. 2008. Fibromyalgia relapse evaluation and efficacy for durability of meaningful relief (FREEDOM): A 6-month, double-blind, placebo-controlled trial with pregabalin. Pain, 136:419-31.

Färber L, Stratz T, Brückle W, et al. 2000. Efficacy and tolerability of tropisetron in primary fibromyalgia - a highly selective and competitive 5-HT3 receptor antagonist. German Fibromyalgia Study Group. Scand J Rheumatol, (Suppl 113):49-54.

Field MJ, Oles RJ, Singh L. 2001. Pregabalin may represent a novel class of anxiolytic agents with a broad spectrum of activity. Br J Pharmacol, 132:1-4.

Field MJ, Cox PJ, StottE, et al. 2006. Identification of the alpha2-delta-1 subunit of voltage-dependent calcium channels as a molecular target for pain mediating the analgesic actions of pregabalin. Proc Natl Acad Sci USA, 103:17537-42.

Frampton JE, Foster RH. 2005. Pregabalin: in the treatment of postherpetic neuralgia. Drugs, 65:111-8.

Freiman TM, Kukolja J, Heinemeyer J, et al. 2001. Modulation of K+evoked $[3 \mathrm{H}]$-noradrenaline release from rat and human brain slices by gabapentin: involvement of KATP channels. Naunyn Schmiedebergs Arch Pharmacol, 363:537-42.

Gendreau RM, Thorn MD, Gendreau JF, et al. 2005. Efficacy of milnacipran in patients with fibromyalgia. $J$ Rheumatol, 32:1975-85.

Goldenberg DL. 2007. Pharmacological treatment of fibromyalgia and other chronic musculoskeletal pain. Best Pract Res Clin Rheumatol, 21:499-511.

Goldenberg DL, Burckhardt C, Crofford L. 2004. Management of fibromyalgia syndrome. JAMA, 292: 2388-95.

Gray P. 2007. Pregabalin in the management of central neuropathic pain. Expert Opin Pharmacother, 8: 3035-41.

Griffiths RR, Johnson MW. 2005. Relative abuse liability of hypnotic drugs: a conceptual framework and algorithm for differentiating among compounds. J Clin Psychiatry, 66 ( Suppl) 9:31-41.

Gürsoy S, Erdal E, Herken H, et al. 2003. Significance of catecholO-methyltransferase gene polymorphism in fibromyalgia syndrome. Rheumatol Int, 23:104-7. 
Henriksson KG, Sorensen J. 2002. The promise of N-methyl-D-aspartate receptor antagonists in fibromyalgia. Rheum Dis Clin North Am, 28:343-51.

Holman AJ, Myers RR. 2005. A randomized, double-blind, placebo-controlled trial of pramipexole, a dopamine agonist, in patients with fibromyalgia receiving concomitant medications. Arthritis Rheum, 52:2495-505.

Kosinski M, Keller SD, Ware JE Jr, et al. 1999. The SF-36 Health Survey as a generic outcome measure in clinical trials of patients with osteoarthritis and rheumatoid arthritis: relative validity of scales in relation to clinical measures of arthritis severity. Med Care, 37(5 Suppl 5):MS23-39.

Lyrica. Drug Information. [on line]. Accessed 18 February. URL: http:// dailymed.nlm.nih.gov/dailymed.drugInfo.cfm?id=5166.

McBeth J, Jones K. 2007. Epidemiology of chronic musculoskeletal pain. Best Pract Res Clin Rheumatol, 21:403-25.

McClelland D, Evans RM, Barkworth L, et al. 2004. A study comparing the actions of gabapentin and pregabalin on the electrophysiological properties of cultured DRG neurones from neonatal rats. BMC Pharmacol, 4:14.

Mease P. 2005. Fibromyalgia syndrome: review of clinical presentation, pathogenesis, outcome measures, and treatment. J Rheumatol, 32(Suppl 75):6-21.

Mease PJ, Russell IJ, Arnold LM, et al. 2008. A randomized, double-blind, placebo-controlled, phase III trial of pregabalin in the treatment of patients with fibromyalgia. $J$ Rheumatol, 35:502-14.

Merck. Manual for Healthcare professionals [online]. Accessed April 27, 2008. URL: www.merck.com/mmpe/sec04/ch040c.html.

MICROMEDEX ${ }^{\circledR}$ Healthcare Series. Pregabalin.

Nørregaard J, Volkmann H, Danneskiold-Samsøe B. 1995. A randomized controlled trial of citalopram in the treatment of fibromyalgia. Pain, 61:445-9.

Owen RT. 2007. Pregabalin: Its efficacy, safety and tolerability profile in fibromyalgia syndrome. Drugs Today, 43:857-63.

Pongratz DE, Sievers M. 2000. Fibromyalgia-symptom or diagnosis: a definition of the position. Scand J Rheumatol, (Suppl 113):3-7.

Randinitis EJ, Posvar EL, Alvey CW, et al. 2003. Pharmacokinetics of pregabalin in subjects with various degrees of renal function. $J$ Clin Pharmacol, 43:277-83.

Rooks DS. 2007. Fibromyalgia treatment update. Curr Opin Rheumatol, 19:111-7.

Russell AS. 1999. Effect of gamma-hydroxybutyrate on pain, fatigue, and alpha sleep anomaly in patients with fibromyalgia. $J$ Rheumatol, 26:2712.

Russell IJ, Orr MD, Littman B, et al. 1994. Elevated cerebrospinal fluid levels of substance $\mathrm{P}$ in patients with the fibromyalgia syndrome. Arthritis Rheum, 37:1593-601.

Scharf MB, Baumann M, Berkowitz DV. 2003.The effects of sodium oxybate on clinical symptoms and sleep patterns in patients with fibromyalgia. J Rheumatol, 30:1070-4.
Selak I. 2001. Pregabalin (Pfizer). Curr Opin Investig Drugs, 2: 828-34.

Senay EC, Adams EH, Geller A, et al. 2003. Physical dependence on Ultram (tramadol hydrochloride): both opioid-like and atypical withdrawal symptoms occur. Drug Alcohol Depend, 69:233-41.

Sills GJ. 2006. The mechanisms of action of gabapentin and pregabalin. Curr Opin Pharmacol, 6:108-13.

Späth M, Stratz T, Färber L, et al. 2004. Treatment of fibromyalgia with tropisetron - dose and efficacy correlations. Scand J Rheumatol, (Suppl 119):63-6.

Staud R, Vierck CJ, Robinson ME, et al. 2005. Effects of the N-methyl-Daspartate receptor antagonist dextromethorphan on temporal summation of pain are similar in fibromyalgia patients and normal control subjects. J Pain, 6:323-32.

Staud R. 2006. Biology and therapy of fibromyalgia: pain in fibromyalgia syndrome. Arthritis Res Ther, 8:208.

Staud R, Price DD. 2008. Long-term trials of pregabalin and duloxetine for fibromyalgia symptoms: How study designs can affect placebo factors. Pain, 136:232-4.

Vartanian MG, Radulovic LL, Kinsora JJ, et al. 2006. Activity profile of pregabalin in rodent models of epilepsy and ataxia. Epilepsy Res, 68:189-205.

Ware JE, Sherbourne CD. 1992. The MOS 36-item short-form health survey (SF-36). I. Conceptual framework and item selection. Med Care, 30:473-83.

White KP, Nielson WR, Harth M, et al. 2002. Chronic widespread musculoskeletal pain with or without fibromyalgia: psychological distress in a representative community adult sample. J Rheumatol, 29:588-94.

Willmore LJ. 2000. Clinical pharmacology of new antiepileptic drugs. Neurology, 55(11 Suppl 3):S17-24.

Wolfe F, Smythe HA, Yunus MB, et al. 1990. The American College of Rheumatology 1990 Criteria for the Classification of Fibromyalgia. Report of the Multicenter Criteria Committee. Arthritis Rheum, 33:160-72.

Wolfe F, Cathey MA, Hawley DJ. 1994. A double-blind placebo controlled trial of fluoxetine in fibromyalgia. Scand J Rheumatol, 23:255-9.

Wolfe F, Ross K, Anderson J, et al. 1995. The prevalence and characteristics of fibromyalgia in the general population. Arthritis Rheum, 38:19-28

Wu SC, Wrobel JS, Armstrong DG. 2007. Assessing the impact of pharmacologic intervention on the quality of life in diabetic peripheral neuropathic pain and fibromyalgia. Pain Med, 8(Suppl 2): S33-42.

Zareba G. 2007. Pregabalin: a new agent for the treatment of neuropathic pain. Drugs Today, 41:509-16. 
